Research Article

\title{
Ginsenoside Rg3 Attenuates Angiotensin II-Mediated Renal Injury in Rats and Mice by Upregulating Angiotensin-Converting Enzyme 2 in the Renal Tissue
}

\author{
Hui Liu, ${ }^{1}$ Yichuan Jiang $\mathbb{D}^{1},{ }^{1}$ Min Li, ${ }^{1}$ Xiaofeng Yu $\mathbb{D},{ }^{1}$ Dayun Sui $\mathbb{D},{ }^{1}$ and Li Fu $\mathbb{D}^{2,3}$ \\ ${ }^{1}$ Department of Pharmacology, School of Pharmaceutical Sciences, Jilin University, Changchun 130021, China \\ ${ }^{2}$ Jilin Yatai Pharmaceutical Co., Ltd., Changchun 130033, China \\ ${ }^{3}$ Institute of Dalian Fusheng Natural Medicine, Dalian 116600, China
}

Correspondence should be addressed to Dayun Sui; suidayun@hotmail.com and Li Fu; dlfulii@aliyun.com

Received 28 April 2019; Revised 5 October 2019; Accepted 24 October 2019; Published 29 November 2019

Academic Editor: Raffaele Pezzani

Copyright (C) 2019 Hui Liu et al. This is an open access article distributed under the Creative Commons Attribution License, which permits unrestricted use, distribution, and reproduction in any medium, provided the original work is properly cited.

Angiotensin II- (Ang II-) mediated renal injury represents a major pathogenetic mechanism in most chronic kidney diseases. Our previous research demonstrated that ginsenoside $\operatorname{Rg} 3(\mathrm{Rg} 3)$ attenuates Ang II elevation in the myocardium in spontaneously hypertensive rats (SHR). It is possible that $\mathrm{Rg} 3$ has similar effects in the renal tissue. In this research, we first demonstrated that Rg3 could attenuate Ang II increase in the kidney of SHR and reduce hypertensive nephropathy progression. Then, we found that Rg3 attenuated Ang II increase by upregulating angiotensin-converting enzyme 2 (ACE2) in the renal tissue. We confirmed this finding in an exogenous Ang II-infused mice model of renal injury, and two models showed consistent results. In conclusion, Rg3 attenuates Ang II-mediated renal injury in rats and mice by upregulating ACE2 in the renal tissue. This research is the first to demonstrate that $\mathrm{Rg} 3$ increases tissue ACE2 levels in vivo.

\section{Introduction}

Ginsenoside $\operatorname{Rg} 3$ (Rg3), an uncommon ginsenoside transformed from other ginsenosides during heating [1], shows strong antitumor activity [2-6]. In the industrialized production, chemical [1] or biological $[7,8]$ techniques are employed for Rg3 synthesis. Shenyi Capsule, whose principal component is Rg3, constitutes a common antitumor drug in China, produced by our company (Jilin Yatai Pharmaceutical Co., Ltd.).

Besides its antitumor activity, $\operatorname{Rg} 3$ has also been reported to have cardiovascular protective effects through multiple mechanisms $[6,9,10]$, including antiinflammation, antioxidative stress, and antifibrosis. Our study indicated that Rg3 exerts cardioprotective effects not depending on blood pressure reduction in spontaneously hypertensive rats (SHR), possibly in association with attenuated angiotensin II (Ang II) increase in the myocardium [10]. It is also known that Ang II increase mediates inflammation, oxidative stress, and fibrosis in the myocardium [11], which could be attenuated by $\mathrm{Rg} 3$.

Indeed, elevated Ang II induces not only cardiovascular injury but also kidney damage in SHR [12]. Hypertensive nephropathy represents a common complication of hypertension, and one of its major mechanisms is Ang IIassociated inflammation, oxidative stress, and fibrosis in the renal tissue [13-15]. As the mechanisms of cardiovascular and renal injuries in SHR have common features, it is interesting to assess whether $\mathrm{Rg} 3$ has renal protective effects in SHR. Furthermore, if Rg3 has such effects in SHR and is associated with the attenuation of Ang II increase, an exogenous Ang II infusion mouse model of renal injury [15] could be used to further confirm these results.

\section{Materials and Methods}

2.1. Chemicals and Reagents. Rg3 (95\% purity) provided by Jilin Yatai Pharmaceutical (China) was dissolved in 0.5\% 
sodium carboxymethyl cellulose solution (0.5\% CMC-Na) for use. Ang II (97\% purity) was purchased from SigmaAldrich (USA) and dissolved in physiological saline. The remaining chemicals were of analytical grade.

2.2. Animals and Treatments. A total of $16 \mathrm{Wistar}-\mathrm{Kyoto}$ rats (WKY) and 16 SHR (male, 16 17 weeks old) (Beijing Vital River Laboratory Animal Technology, China) were housed with rodent chow and water ad libitum. Experiments involving animals followed the Guide for the Care and Use of Laboratory Animals of Jilin University, with approval from the institutional Ethics Committee.

The animals were grouped into four: WKY group (8 WKY, orally administered $0.5 \%$ CMC-Na); SHR group (8 SHR, orally administered $0.5 \% \mathrm{CMC}-\mathrm{Na}) ; W K Y+R g 3$ group (8 WKY, orally administered $20 \mathrm{mg} \cdot \mathrm{kg}^{-1} \cdot \mathrm{d}^{-1} \mathrm{Rg} 3$ ); SHR $+R g 3$ group (8 SHR, orally administered $20 \mathrm{mg} \cdot \mathrm{kg}^{-1} \cdot \mathrm{d}^{-1} \mathrm{Rg} 3$ ).

$\mathrm{Rg} 3$ or placebo administration was carried out once daily for 42 days. This was followed by animal sacrifice and blood and renal tissue sample collection. The renal tissue specimens underwent fixation with $4 \%$ formalin (histopathology) or were snap-frozen with liquid nitrogen and kept at $-80^{\circ} \mathrm{C}$ (reverse transcription quantitative real-time polymerase chain reaction (RT-qPCR) and enzyme-linked immunosorbent assay (ELISA)).

A total of 24 C57BL/6 mice (male, 10 weeks old) (Beijing Vital River Laboratory Animal Technology) were maintained with rodent chow and water at will. Experiments involving animals followed the Guide for the Care and Use of Laboratory Animals of Jilin University, with approval from the institutional Ethics Committee.

Subcutaneous implantation of a 1002 osmotic minipump (Alza, USA) was performed at the dorsum of the neck for Ang II $\left(1.5 \mathrm{mg} \cdot \mathrm{kg}^{-1} \cdot \mathrm{d}^{-1}\right)$ or normal saline infusion [15]. The animals were assigned to four groups: Saline group (4 mice, infused with normal saline and orally administered $0.5 \%$ CMC-Na); Ang II group (4 mice, infused with Ang II and orally administered $0.5 \%$ CMC-Na); Saline $+R g 3$ group (4 mice, infused with normal saline and orally administered $20 \mathrm{mg} \cdot \mathrm{kg}^{-1} \cdot \mathrm{d}^{-1} \mathrm{Rg} 3$ ); Ang $I I+R g 3$ group (4 mice, infused with Ang II and orally administered $\left.20 \mathrm{mg} \cdot \mathrm{kg}^{-1} \cdot \mathrm{d}^{-1} \mathrm{Rg} 3\right)$.

$\mathrm{Rg} 3$ or placebo administration was performed daily for 14 days. This was followed by animal sacrifice and blood and renal tissue sample collection. The renal tissue specimens underwent fixation with $4 \%$ formalin (histopathology) or were snap-frozen with liquid nitrogen and kept at $-80^{\circ} \mathrm{C}$ (RT-qPCR and ELISA).

2.3. Blood Pressure Assessment. Systolic (SBP) and diastolic (DBP) blood pressure measurements in rats and mice were performed by the tail-cuff technique using a small animal sphygmomanometer (BP-2010A; Softron Biotechnology, China) [16] on the initial and final days of treatment (6 and 2 weeks in rats and mice, respectively).

2.4. Serum Creatinine and Blood Urea Nitrogen (BUN) Level Assessment. Blood specimens were submitted to centrifugation $\left(1500 \mathrm{~g}, 4^{\circ} \mathrm{C}\right.$ for $\left.15 \mathrm{~min}\right)$, and the resulting serum was kept at $-80^{\circ} \mathrm{C}$ for biochemical assays. Creatinine assay and BUN assay kits were purchased form Nanjing Jiancheng Bioengineering Institute (China), and creatinine and BUN levels were assayed in accordance with the manufacturer's protocols.

2.5. Histopathological Assessment. Renal tissue specimens underwent fixation with $4 \%$ formalin, paraffin embedding, sectioning at $4 \mu \mathrm{m}$, and staining with hematoxylin and eosin (H\&E) and Masson trichrome stain, respectively. Then, a Nikon E100 light microscope (Nikon, Japan) was employed for analysis.

2.6. Immunohistochemistry (IHC). Antiangiotensin-converting enzyme (ACE) and antiangiotensin-converting enzyme 2 (ACE2) primary antibodies were produced by Bioss Antibodies (China). Peroxidase-linked goat anti-rabbit secondary antibodies and the DAB and two-step rabbit IHC kits were provided by ZSGB-BIO (China). IHC was carried out as proposed by ZSGB-BIO. Photomicrographs were acquired and assessed by Image Pro Plus 6.0 (Media Cybernetics, USA).

2.7. Ang II and Angiotensin 1-7 (Ang 1-7) Level Assessment in the Renal Tissue. The supernatants of renal specimens were prepared as follows. A total of $100 \mathrm{mg}$ of the renal tissue was homogenized in $900 \mu \mathrm{L}$ of ice-cold normal saline and submitted to centrifugation $\left(1000 \mathrm{~g}, 4^{\circ} \mathrm{C}\right.$ for $\left.15 \mathrm{~min}\right)$. The resulting supernatants were kept at $-80^{\circ} \mathrm{C}$ until analysis with Ang II and Ang 1-7 ELISA kits (Cusabio Biotech, China), respectively, in accordance with the manufacturer's protocols.

2.8. RNA Purification and RT-qPCR. Total RNA isolation was carried out with TRIzol reagent (Thermo Fisher Scientific, USA) as directed by the manufacturer. Reverse transcription and qPCR were carried out with TransScript Green Two-Step qRT-PCR SuperMix (TransGen Biotech, China) on a Stratagene $\mathrm{Mx} 3000 \mathrm{P}$ (Agilent Technologies, USA) at $94^{\circ} \mathrm{C}(5 \mathrm{sec}$ ), $60^{\circ} \mathrm{C}(15 \mathrm{sec})$, and $72^{\circ} \mathrm{C}(10 \mathrm{sec})$ for 40 cycles. The $2^{-\Delta \Delta \mathrm{Ct}}$ method [17] was employed for analysis, with GAPDH used for normalization. Primers are listed in Table 1.

2.9. Statistical Analysis. SPSS 16.0 (SPSS, USA) was employed for all statistical analyses. Data are mean \pm standard deviation (SD). One-way analysis of variance (ANOVA) with Tukey's post hoc test was employed for group comparisons, with $P<0.05$ indicating statistical significance.

\section{Results}

3.1. Rg3 Attenuates Early Nephropathy in SHR. As we mentioned in a previous report [10], Rg3 had no significant effect on blood pressure. As shown in Figures 1(a) and 1(b), SBP and DBP in the two groups of SHR were markedly elevated in comparison with those of the two groups of WKY 
TABle 1: Primer sequences of GAPDH, TNF- $\alpha$, IL-6, TGF- $\beta 1$, and p47phox.

\begin{tabular}{|c|c|}
\hline $\begin{array}{l}\text { Primer } \\
\text { names }\end{array}$ & Sequences \\
\hline \multicolumn{2}{|l|}{ Rats } \\
\hline \multirow{2}{*}{ GAPDH } & Forward: 5'TTGTCAGCAATGCATCCTGC-3' \\
\hline & Reverse: 5'-CGGCATGTCAGATCCACAAC-3' \\
\hline \multirow{2}{*}{ TNF- $\alpha$} & Forward: 5'-GTCGTAGCAAACCACCAAGC-3' \\
\hline & Reverse: $5^{\prime}$-TGTGGGTGAGGAGCACGTAG-3' \\
\hline \multirow{2}{*}{ IL-6 } & Forward: 5'-TGTATGAACAGCGATGATG-3' \\
\hline & Reverse: $5^{\prime}$-AGAAGACCAGAGCAGATT-3' \\
\hline \multirow{2}{*}{ TGF- $\beta 1$} & $\begin{array}{l}\text { Forward: } 5^{\prime} \text {-ACCTGCAAGACCATCGACATG- } \\
\qquad 3^{\prime}\end{array}$ \\
\hline & $\begin{array}{c}\text { Reverse: } 5^{\prime} \text {-CGAGCCTTAGTTTGGACAGGAT- } \\
3^{\prime}\end{array}$ \\
\hline \multirow{2}{*}{ p47phox } & Forward: 5'-GCACTGAAAGGCGGTCCTAT-3' \\
\hline & Reverse: $5^{\prime}$-TACCCGTGGAGAGAAACCCA-3' \\
\hline \multicolumn{2}{|l|}{ Mice } \\
\hline \multirow{3}{*}{ GAPDH } & Forward: $5^{\prime}-$ \\
\hline & CССТTCATTGACCTCAACTACATG-3' \\
\hline & Reverse: 5'-CTTCTCCATGGTGGTGAAGAC-3' \\
\hline \multirow{2}{*}{ TNF- $\alpha$} & Forward: 5'-GTCGTAGCAAACCACCAAGT-3' \\
\hline & Reverse: $5^{\prime}$-TGTGGGTGAGGAGCACGTAG-3' \\
\hline \multirow[t]{2}{*}{ IL-6 } & $\begin{array}{c}\text { Forward: } 5^{\prime}- \\
\text { GTCCTTCAGAGAGATACAGAAACT-3' } \\
\text { Reverse: } 5^{\prime} \text { - }\end{array}$ \\
\hline & AGCTTATCTGTTAGGAGAGCATTG-3' \\
\hline \multirow[b]{2}{*}{ TGF- $\beta 1$} & $\begin{array}{c}\text { Forward: } 5^{\prime} \text {-ACCTGCAAGACCATCGACATG- } \\
3^{\prime}\end{array}$ \\
\hline & Reverse: $5^{\prime}$-CGAGCCTTAGTTTGGACAGGAT- \\
\hline \multirow{2}{*}{ p47phox } & Forward: 5'-ACCTGTCGGAGAAGGTGGT-3' \\
\hline & 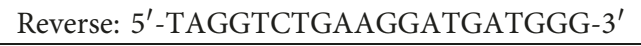 \\
\hline
\end{tabular}

before treatment. Similar findings were obtained after the 6week treatment. In addition, blood pressure in neither WKY nor SHR was changed by Rg3 treatment.

Similar to the cardioprotective effects of Rg3 in SHR, Rg3 exerted renal protective effects independent of blood pressure reduction. Kidney H\&E- and Masson-stained sections in the $W K Y+R g 3$ group showed the same features found in the WKY group. Renal sections of SHR showed congestion of glomerular capillaries and tubulointerstitial fibrosis based on Masson staining; $\operatorname{Rg} 3$ could attenuate these changes in glomerular capillaries and tubules (Figure 2).

The histopathological changes in kidneys of SHR were at the early stage in this study. This finding was confirmed by serum creatinine and BUN levels (Figures 1(c) and 1(d)). Creatinine and BUN levels in the two groups of SHR were somewhat elevated in comparison with those of the two groups of WKY, but the differences were not significant. The $S H R$ and $S H R+R g 3$ groups also showed comparable values.

These results indicated that hypertension-associated nephropathy in SHR was at the compensation stage. Rg3 could attenuate early nephropathy in SHR and might be able to prevent it from progressing into the decompensation stage.

3.2. Rg3 Reduces Ang II Levels in the Renal Tissue in SHR. There are two main angiotensin-converting enzymes which regulate the levels of Ang II in vivo, including ACE and
ACE2 [18, 19]. ACE transforms angiotensin I into Ang II, and ACE2 transforms Ang II into Ang 1-7. So upregulation of ACE induces Ang II increase, while ACE2 upregulation attenuates Ang II increase.

According to ELISA data (Figure 3), Ang II levels in the renal tissue of the SHR group were significantly higher than those of $W K Y$ and $W K Y+R g 3$ groups. Treatment with $\mathrm{Rg} 3$ significantly reduced Ang II levels in the renal tissue in SHR, while showing limited effects in the WKY group. Ang 1-7 amounts in the SHR group were significantly higher than those of the $W K Y$ and $W K Y+R g 3$ groups, with the $S H R+R g 3$ showing even significantly higher values compared with the $S H R$ group.

IHC results (Figure 4) showed that ACE levels were markedly elevated in the two SHR groups compared with the two WKY groups. ACE2 amounts in the renal tissue were compensatorily upregulated in the $S H R$ group, compared with the $W K Y$ and $W K Y+R g 3$ groups. Meanwhile, $\operatorname{Rg} 3$ treatment could further upregulate ACE2 in the renal tissue in SHR. This was the main mechanism that $\mathrm{Rg} 3$ treatment downregulated Ang II and upregulated Ang 1-7 in the kidneys of SHR. Meanwhile, Rg3 had no significant effect on ACE expression in either WKY or SHR.

3.3. Rg3 Attenuates Inflammation, Oxidative Stress, and Fibrosis in the Renal Tissue of SHR. It was reported that high Ang II levels in the renal tissue cause inflammation, oxidative stress, and fibrosis [15]. The mechanisms include inflammatory factor release, upregulated nicotinamide adenine dinucleotide phosphate (NADPH) oxidase, and activation of the TGF- $\beta 1 / \mathrm{Smad}$ signaling pathway. These changes also occurred in SHR.

RT-qPCR data (Figure 5) demonstrated the relative mRNA amounts of TNF- $\alpha$, IL-6, p47phox (NADPH oxidase subunits), and TGF- $\beta 1$ in the renal tissue in the SHR group were remarkably elevated compared with those of the two WKY groups. The upregulation mediated by Ang II could be inhibited by $\mathrm{Rg} 3$ treatment, as the levels of these four mRNAs in the $S H R+R g 3$ group were significantly lower than those of the SHR group, with no significant difference compared with the two WKY groups. These data indicated Rg3 treatment attenuated inflammation, oxidative stress, and fibrosis in the renal tissue in SHR.

3.4. Rg3 Attenuates Ang II-Mediated Kidney Injury in Mice. Ang II infusion by using the osmotic minipump $\left(1.5 \mathrm{mg} \cdot \mathrm{kg}^{-1} \cdot \mathrm{d}^{-1}\right)$ could raise blood pressure and mediate heart and kidney injury in C57BL/6 mice $[11,15]$. In this research, SBP and DBP in the Ang II and Ang II + Rg3 groups were significantly higher than those of the other two groups after the 2-week Ang II infusion. With or without Ang II infusion, treatment with $\mathrm{Rg} 3$ did not change blood pressure in mice (Figures 6(a) and 6(b)).

According to $\mathrm{H} \& \mathrm{E}$ and Masson photomicrographs (Figure 7), histopathological changes in the kidneys of mice infused Ang II were more obvious compared with those of SHR; kidney sections showed glomerular changes, including hemorrhage, shrunken glomeruli, congestion of 


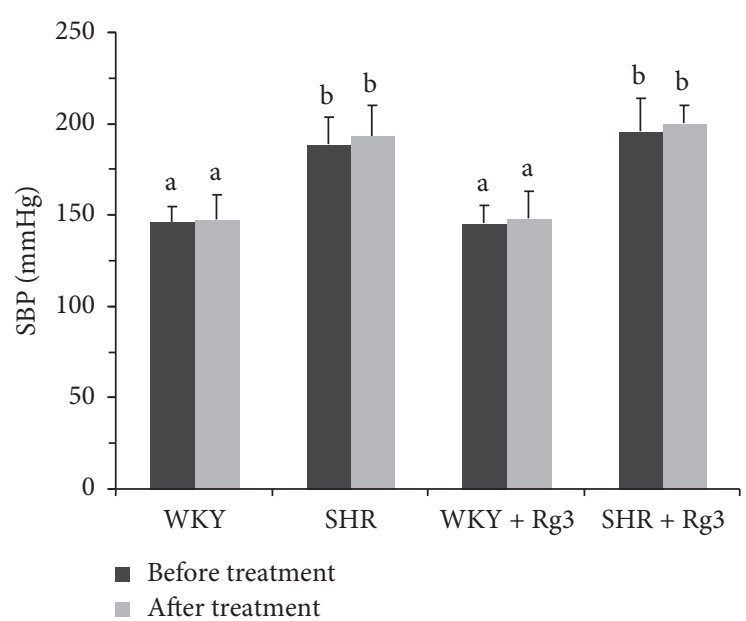

(a)

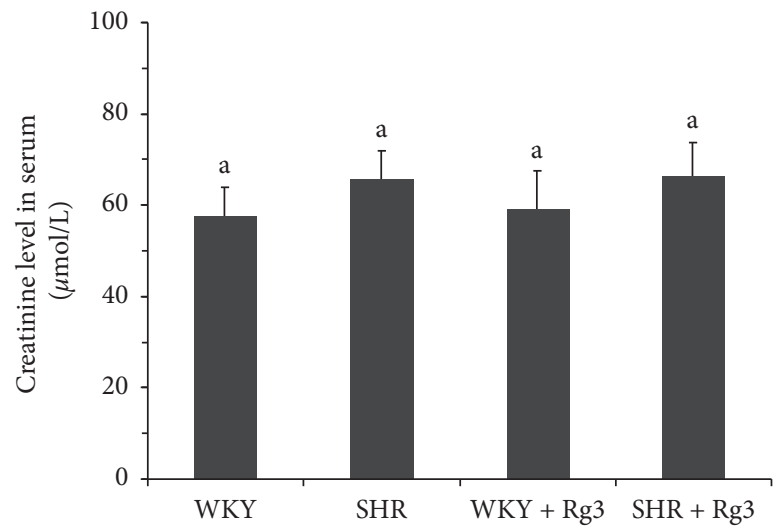

(c)

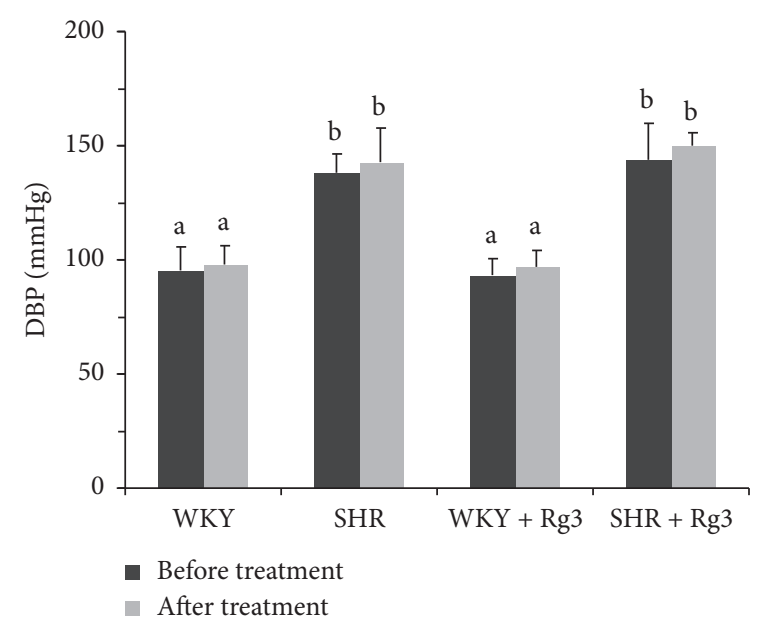

(b)

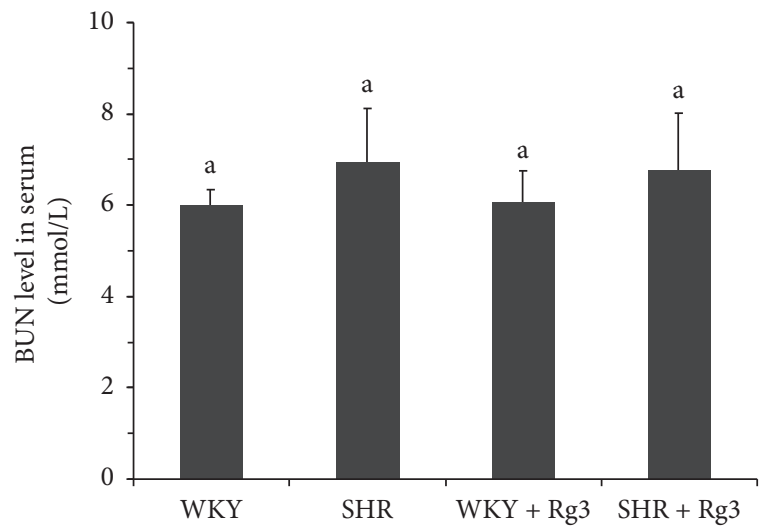

(d)

Figure 1: Blood pressure and serum markers of renal function in rats. SBP (a) and DBP (b) of rats prior to and following 6-week treatment; creatinine (c) and BUN (d) levels in serum in rats. Data are presented as the mean \pm standard deviation, $n=8$. The same superscript letters indicate no significant difference between groups $(P>0.05)$; significant difference existed between groups that do not have the same superscript letter $(P<0.05)$.
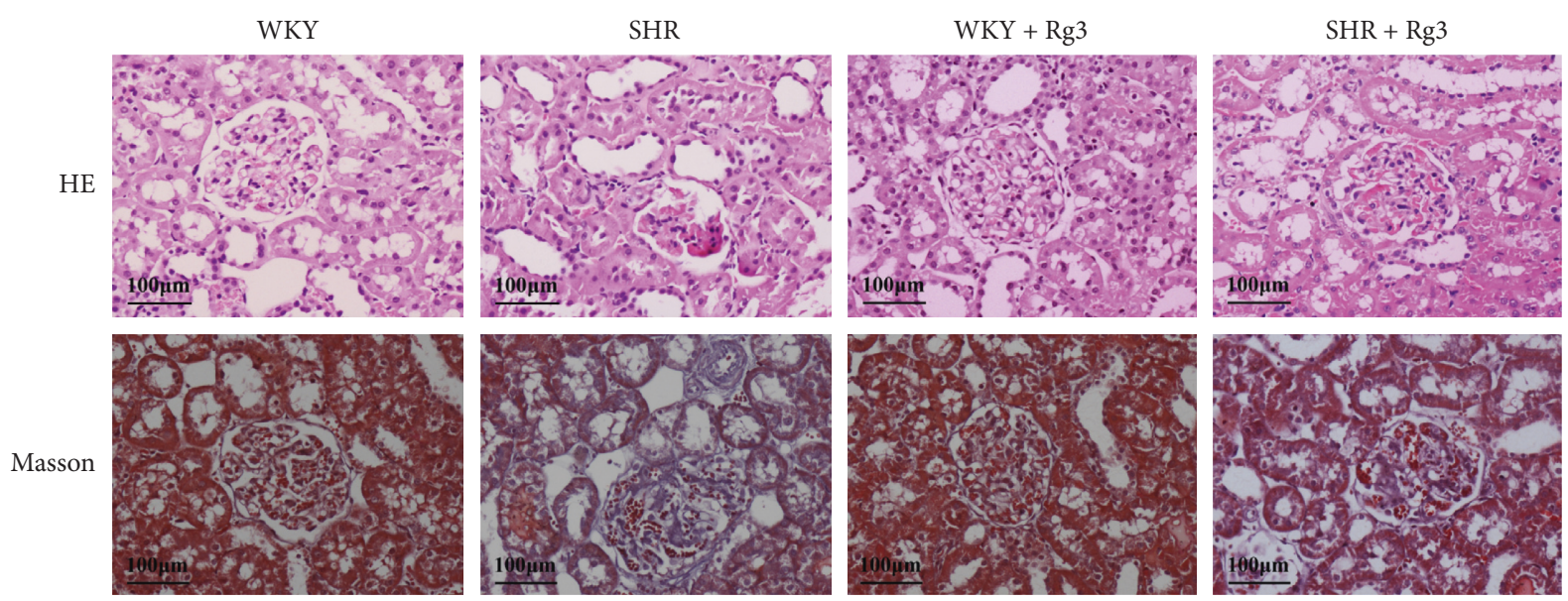

Figure 2: Representative HE and Masson staining histology photomicrographs of the renal tissue in rats.

glomerular capillaries, and severe tubulointerstitial fibrosis in Masson staining. Rg3 could attenuate these changes in the kidney.
In accordance with histopathological data, creatinine and BUN levels in the Ang II group were markedly elevated compared with those of the other three groups, suggesting 


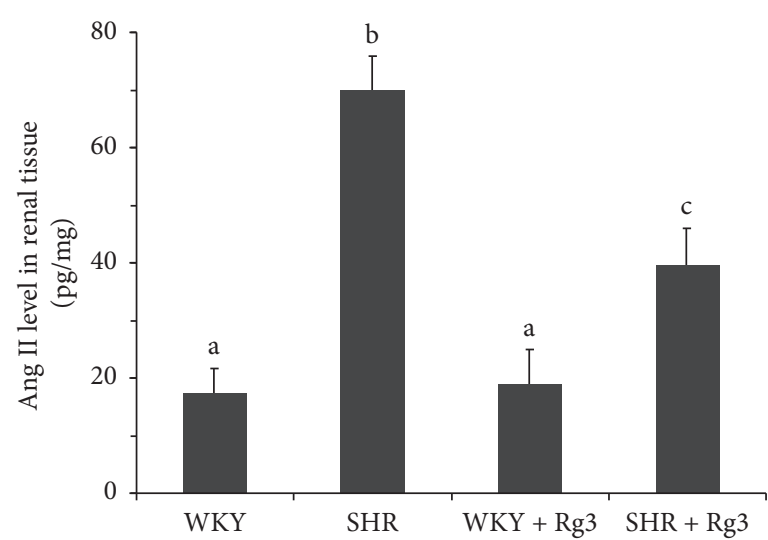

(a)

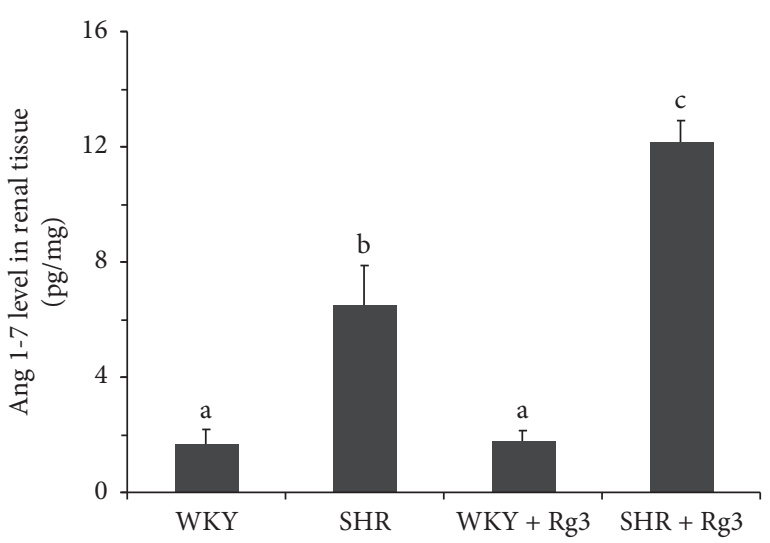

(b)

Figure 3: Angiotensin levels in the renal tissue in rats. (a) Ang II levels in renal tissue; (b) Ang 1-7 levels in renal tissue. Data are presented as the mean \pm standard deviation, $n=8$. The same superscript letters indicate no significant difference between groups $(P>0.05)$; significant difference existed between groups that do not have the same superscript letter $(P<0.05)$.
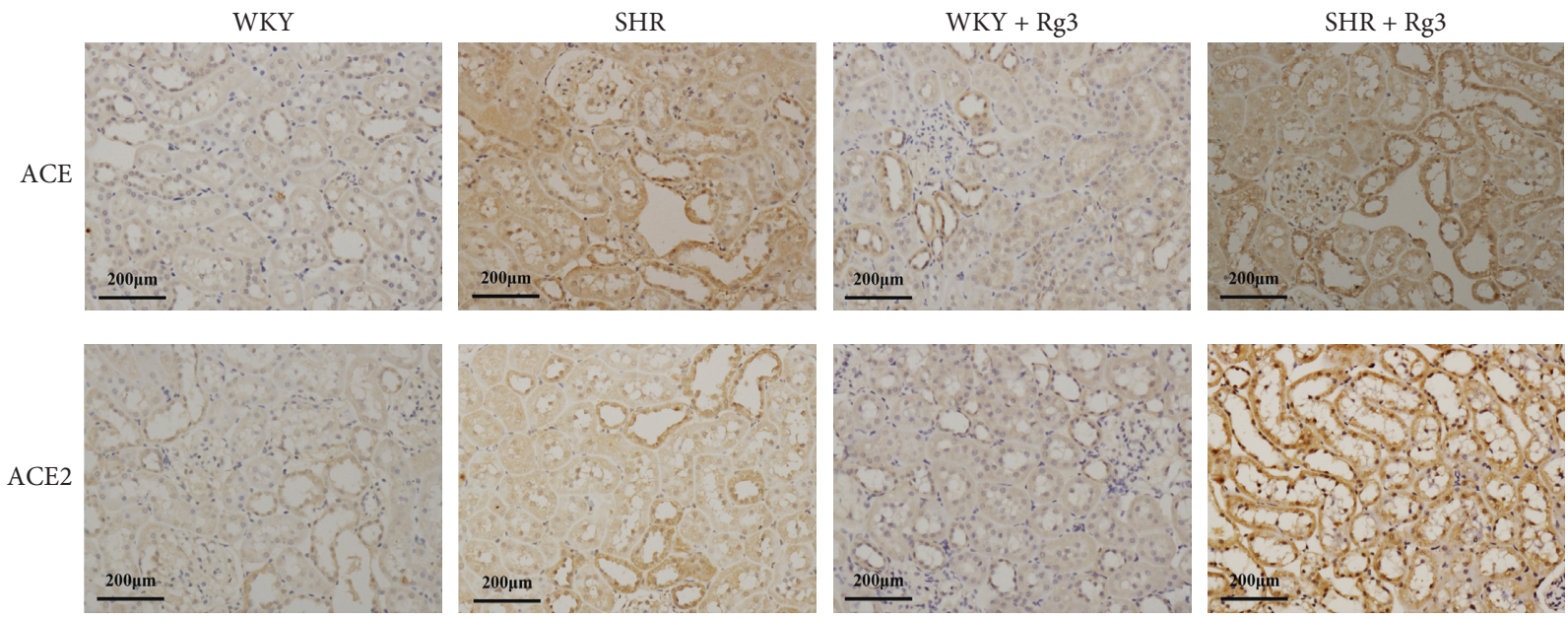

(a)

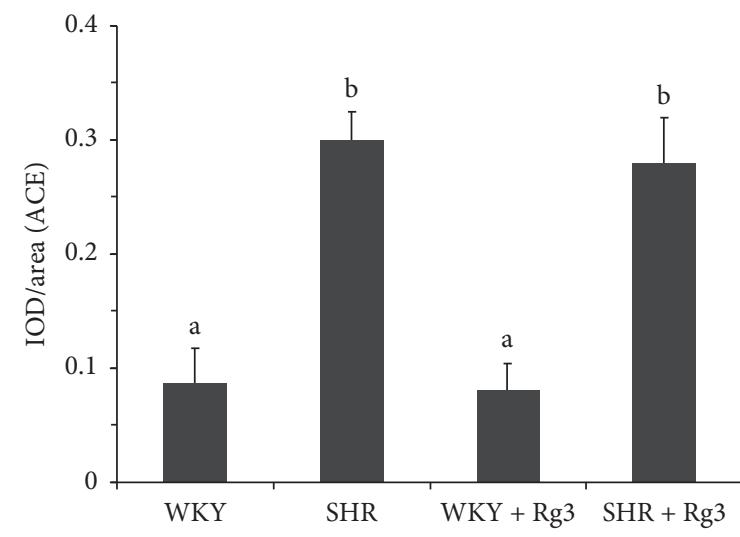

(b)

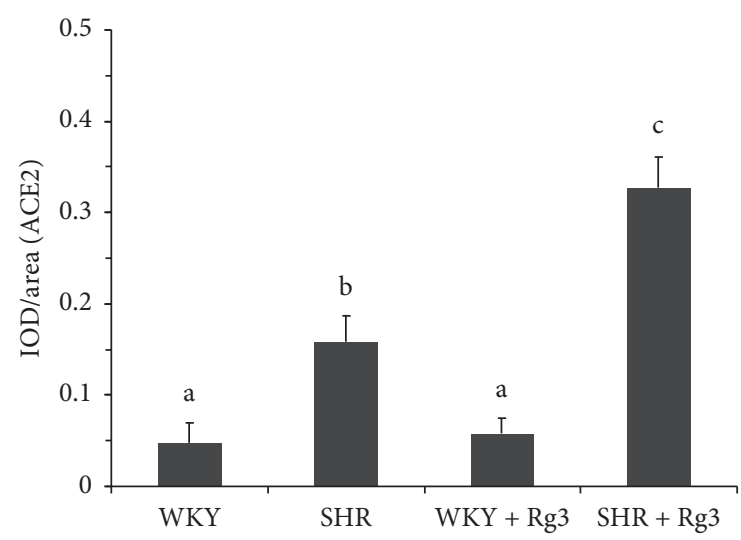

(c)

Figure 4: Levels of the angiotensin-converting enzymes in the renal tissue in rats. (a) Representative IHC staining photomicrographs of the renal tissue in rats. Quantitative results of IHC staining, which were presented as IOD/area and were proportional to the levels of ACE (b) and ACE2 (c). Data are presented as the mean \pm standard deviation, $n=4$. The same superscript letters indicate no significant difference between groups $(P>0.05)$; significant difference existed between groups that do not have the same superscript letter $(P<0.05)$. 


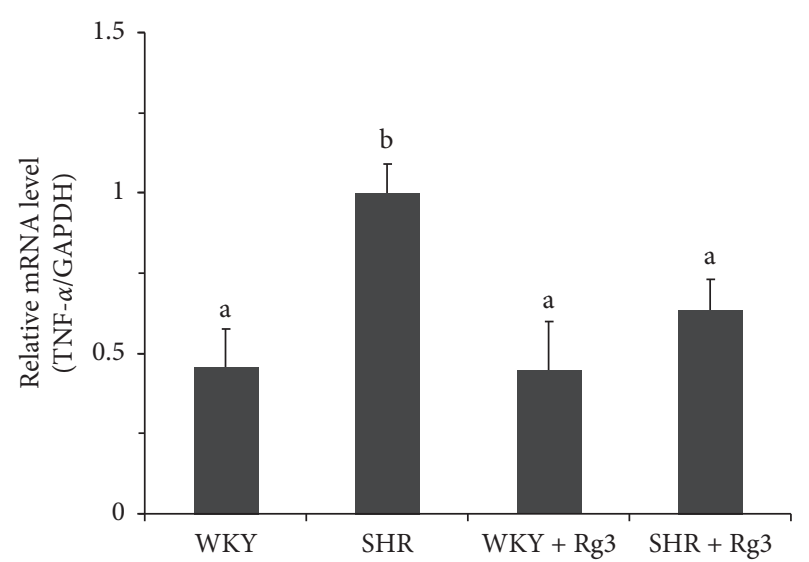

(a)

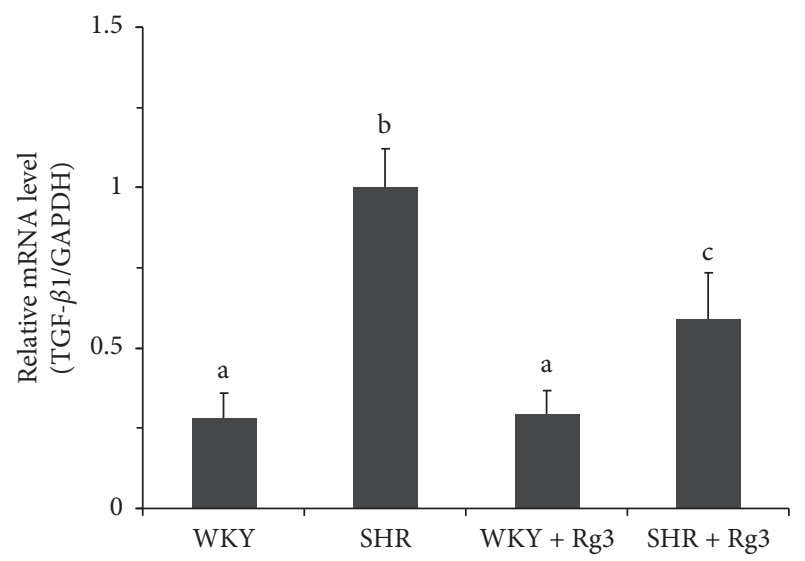

(c)

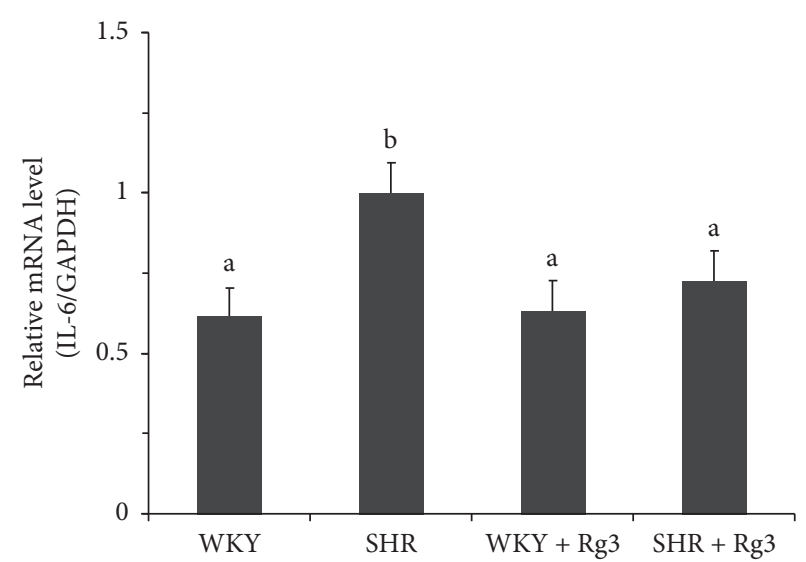

(b)

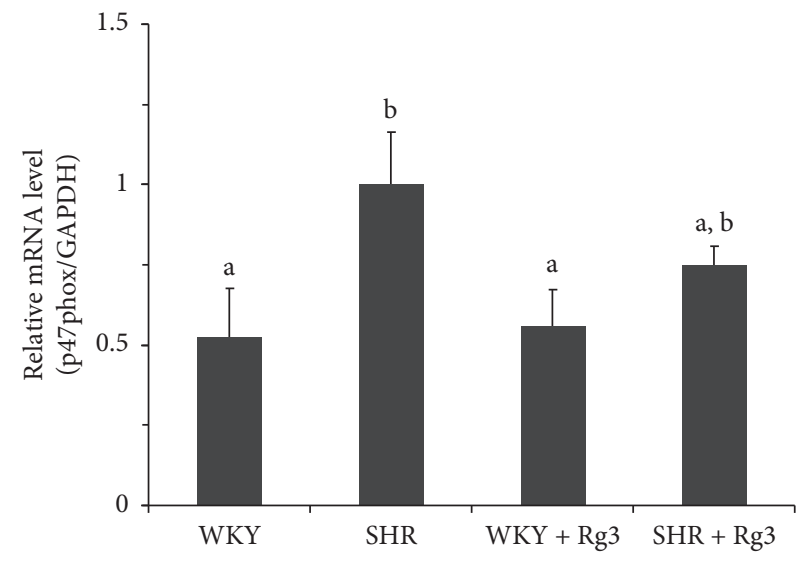

(d)

FIgURE 5: Levels of mRNA associated with inflammation, oxidative stress, and fibrosis in the renal tissue in rats. Relative mRNA levels of TNF- $\alpha$ (a), IL-6 (b), TGF- $\beta 1$ (c), and p47phox (d). GAPDH was used as a housekeeping gene. Data are presented as the mean \pm standard deviation, $n=4$. The same superscript letters indicate no significant difference between groups $(P>0.05)$; significant difference existed between groups that do not have the same superscript letter $(P<0.05)$.

nephropathy in this group was decompensated. Creatinine and BUN levels in the other three groups had no significant differences, indicating $\mathrm{Rg} 3$ treatment improved renal function in mice infused Ang II, and prevented nephropathy progression from compensation to decompensation (Figures 6(c) and 6(d)).

3.5. Rg3 Reduces Ang II Levels in the Renal Tissue in Mice Infused Ang II. According to ELISA data (Figure 8), Ang II infusion significantly raised Ang II levels in the renal tissue in mice, and $\mathrm{Rg} 3$ treatment could attenuate this increase significantly, although Ang II levels in the Ang II + Rg3 group were still significantly higher than those of the two groups infused saline. Meanwhile, Ang 1-7 levels in the Ang II group were significantly higher than those of the Saline and Saline + Rg3 groups; the Ang II $+R g 3$ group showed a significant increase compared with the Ang II group.

IHC data (Figure 9) showed no marked differences in various group pairs in ACE expression. ACE2 expression levels in the Ang $I I+R g 3$ group were markedly elevated compared with those of the other three groups, which might be the main mechanism for degrading exogenous angiotensin.

3.6. Rg3 Attenuates Ang II Associated Inflammation, Oxidative Stress, and Fibrosis in Renal Tissue in Mice. Similar to the changes occurring in SHR, RT-qPCR data (Figure 10) revealed relative TNF- $\alpha$, IL-6, p47phox, and TGF- $\beta 1$ mRNA amounts in the renal tissue in the Ang II group were markedly elevated compared with those of the two groups infused saline. The upregulation mediated by Ang II could be inhibited by $\operatorname{Rg} 3$ treatment, as the levels of these four mRNAs in the Ang $I I+R g 3$ group were significantly lower than those of the Ang II group. These findings indicated Rg3 treatment attenuated inflammation, oxidative stress, and fibrosis caused by Ang II infusion in the renal tissue in mice.

\section{Discussion}

Chronic kidney disease (CKD) represents a growing global health problem [20, 21]. Ang II is a major risk factor for CKD, e.g., hypertensive and diabetic nephropathies. Ang II- 


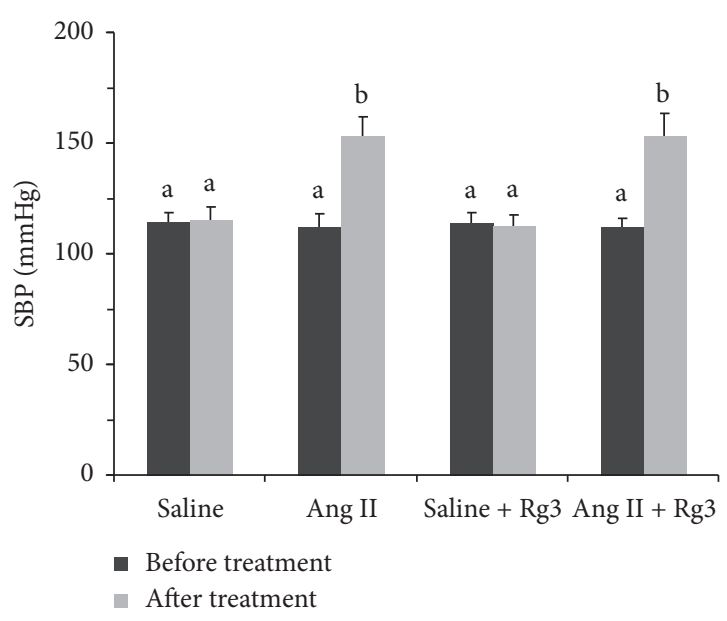

(a)

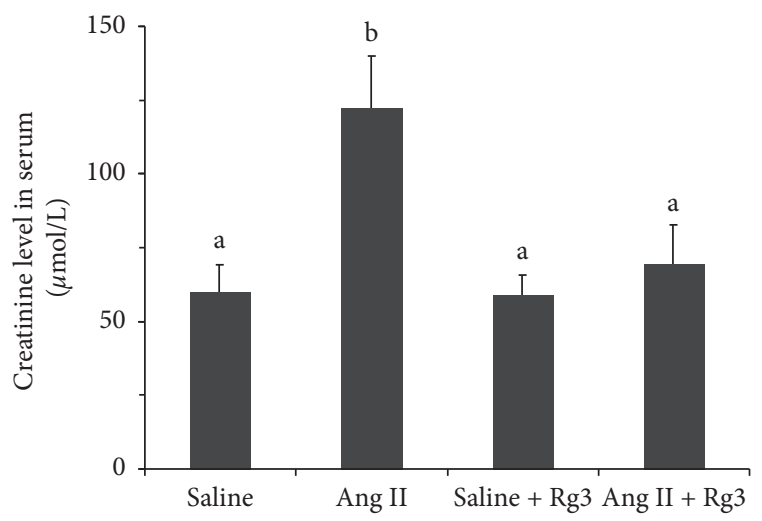

(c)

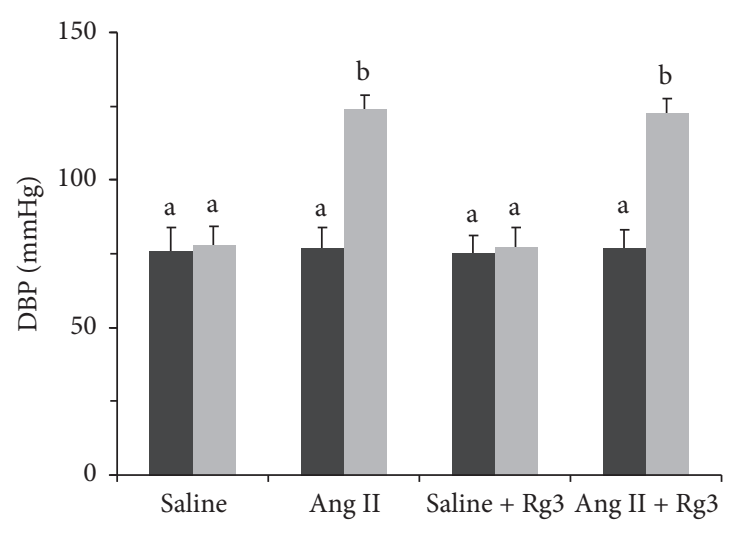

- Before treatment

- After treatment

(b)

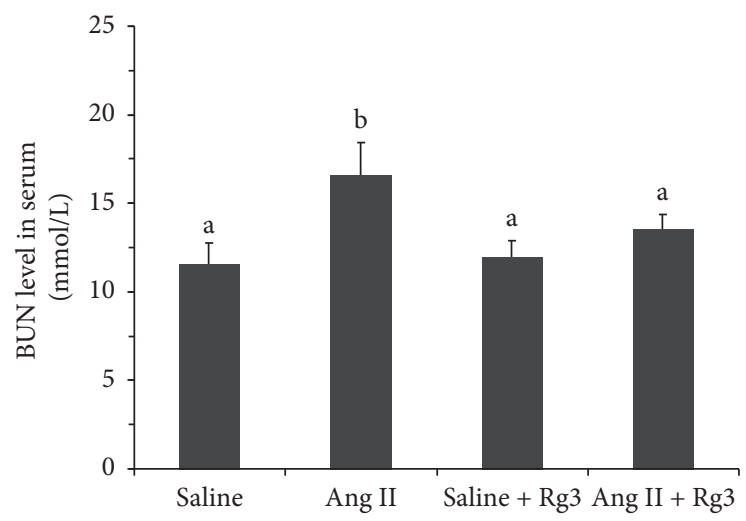

(D)

FIGURE 6: Blood pressure and serum markers of renal function in mice. SBP (a) and DBP (b) of mice prior to and following 2-week treatment; creatinine (c) and BUN (D) levels in serum in mice. Data are presented as the mean \pm standard deviation, $n=4$. The same superscript letters indicate no significant difference between groups $(P>0.05)$; significant difference existed between groups that do not have the same superscript letter $(P<0.05)$.
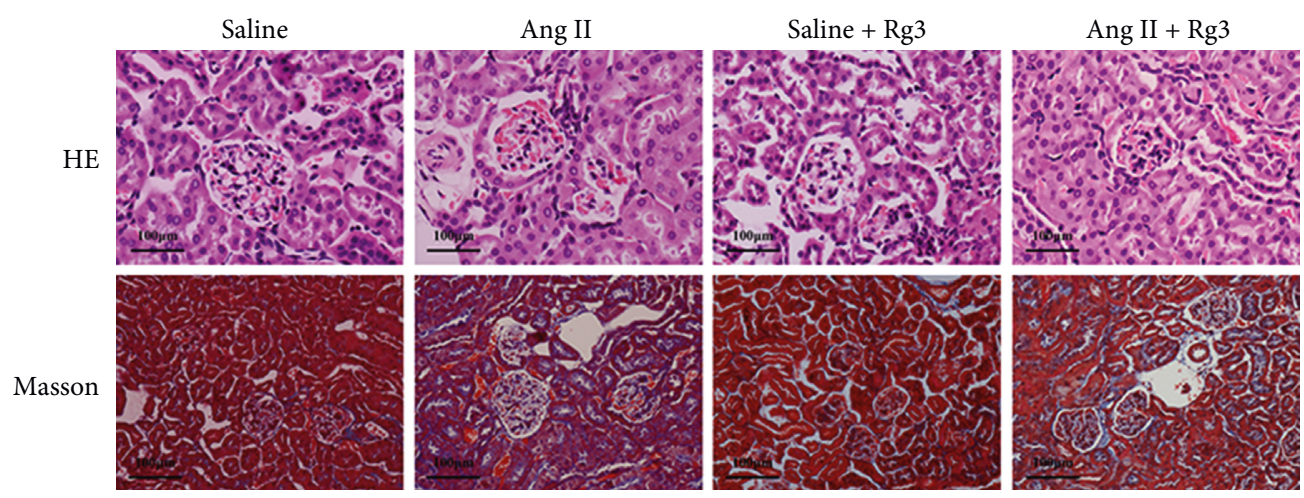

Figure 7: Representative HE and Masson staining histology photomicrographs of the renal tissue in mice.

associated oxidative stress, inflammation, and fibrosis result in reversible or irreversible pathological changes in the renal tissue, which could progress into end-stage renal disease [21].

To counteract Ang II increase in the renal tissue in earlystage hypertensive or diabetic nephropathy, the expression of ACE2 will be compensatorily upregulated. This self- protective mechanism becomes ineffective as the injury is sustained, and hypertensive or diabetic nephropathy would progress into the decompensation stage [19].

It was reported that human recombinant $\mathrm{ACE} 2$ injection could enhance the renal protective effects of ACE2 compensatory upregulation $[15,19]$. These protective effects 


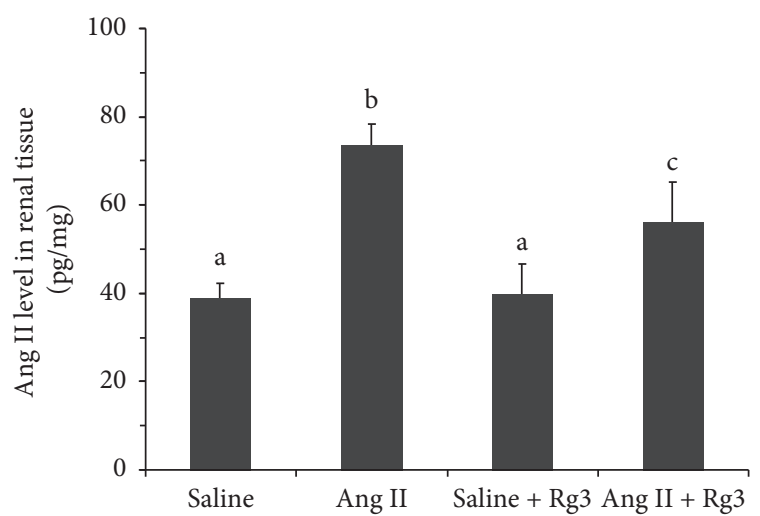

(a)

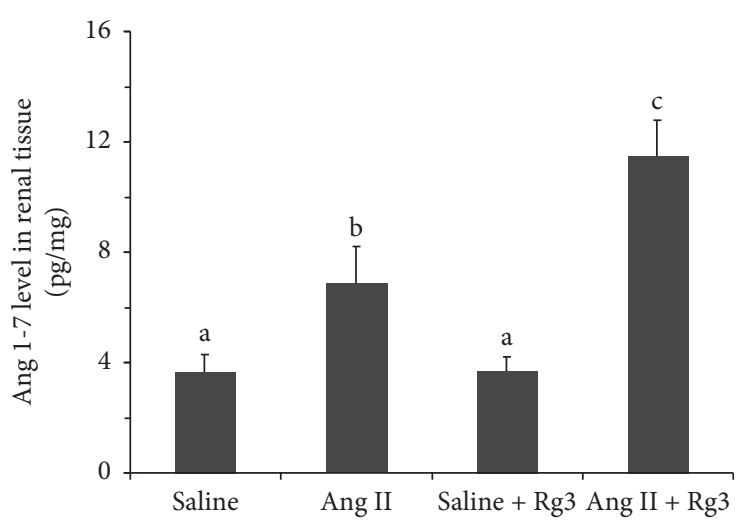

(b)

Figure 8: Angiotensins levels in the renal tissue in mice. Ang II (a) and Ang 1-7 (b) levels in the renal tissue. Data are presented as the mean \pm standard deviation, $n=4$. The same superscript letters indicate no significant difference between groups $(P>0.05)$; significant difference existed between groups that do not have the same superscript letter $(P<0.05)$.
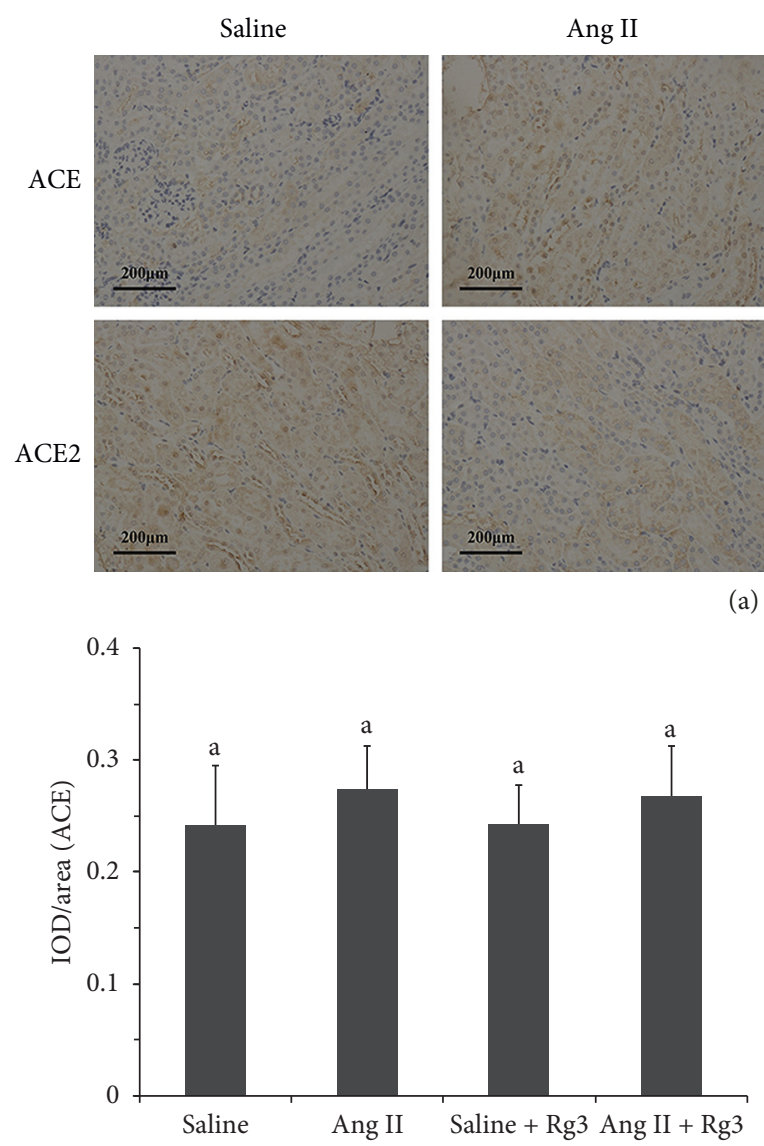

(b)

(a)
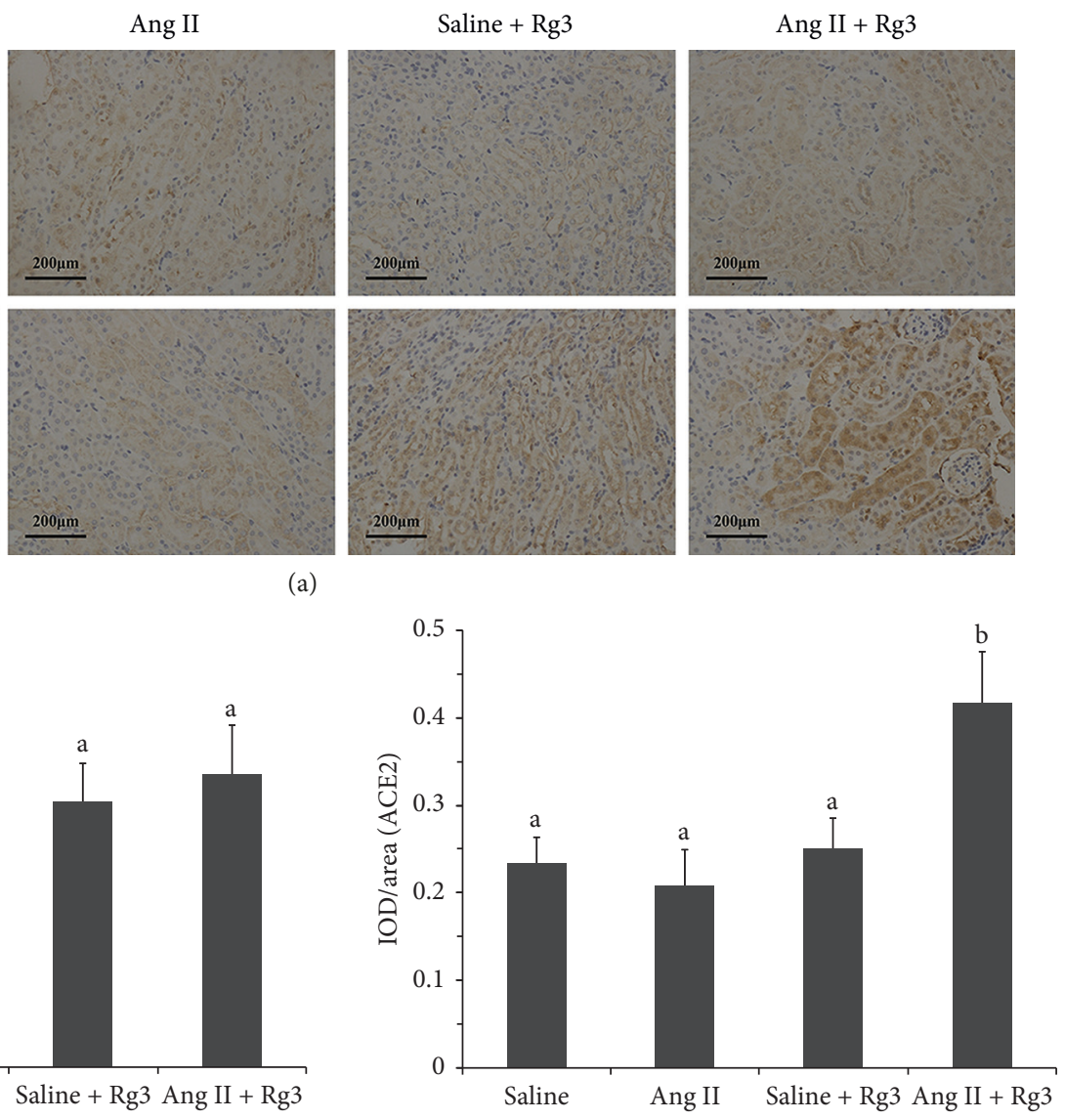

(c)

FIGURE 9: Levels of the angiotensin-converting enzymes in the renal tissue in mice. (a): Representative IHC staining photomicrographs of the renal tissue in mice. Quantitative results of IHC staining, which were presented as IOD/area and were proportional to the levels of ACE (b) and ACE2 (c). Data are presented as the mean \pm standard deviation, $n=4$. The same superscript letters indicate no significant difference between groups $(P>0.05)$; significant difference existed between groups that do not have a same superscript letter $(P<0.05)$.

occur even in ACE2-knockout mice [15]. Therefore, it is available to reduce the progression of nephropathy increasing ACE2 levels in the renal tissue.
Rg3 exerts protective effects on various organs, including the heart, kidney and liver $[6,9,10,22]$. We have demonstrated that the cardioprotective effects of $\mathrm{Rg} 3$ are 


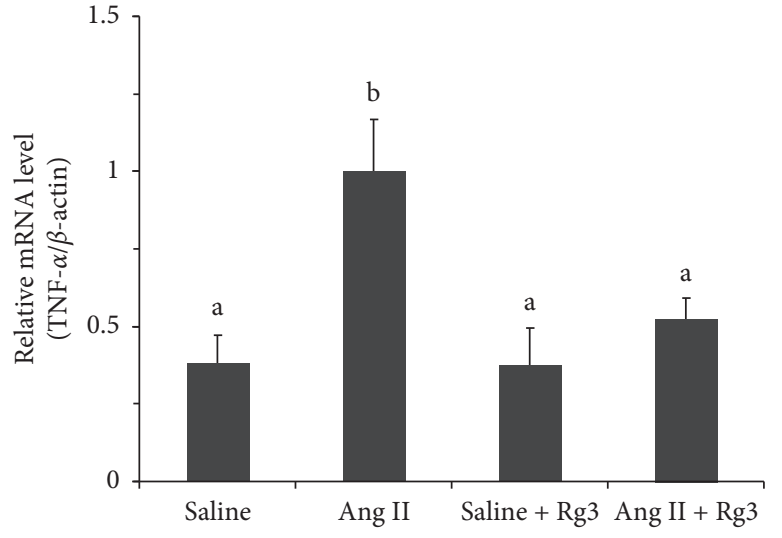

(a)

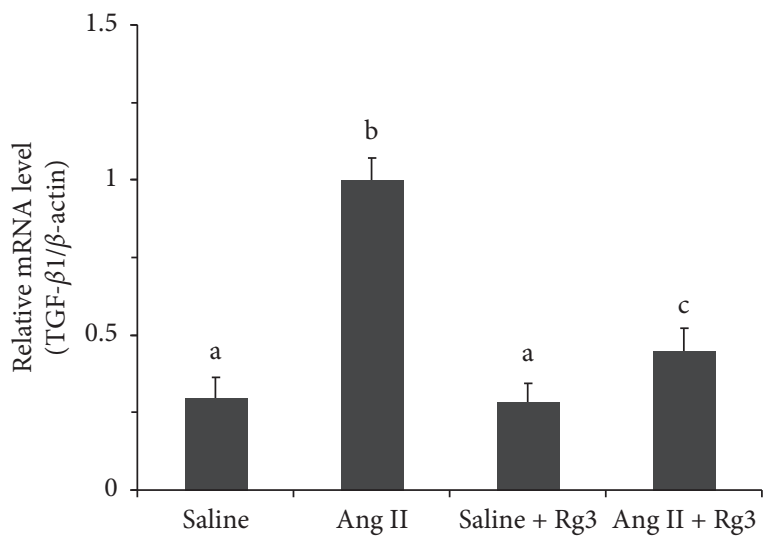

(c)

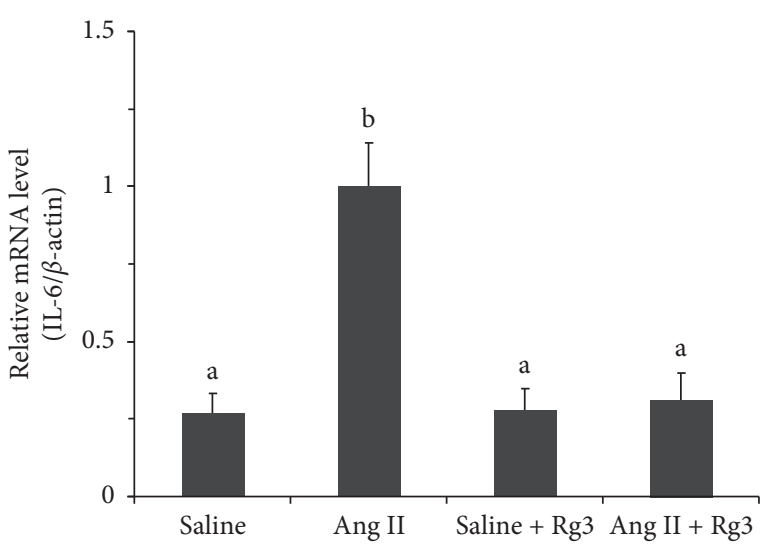

(b)

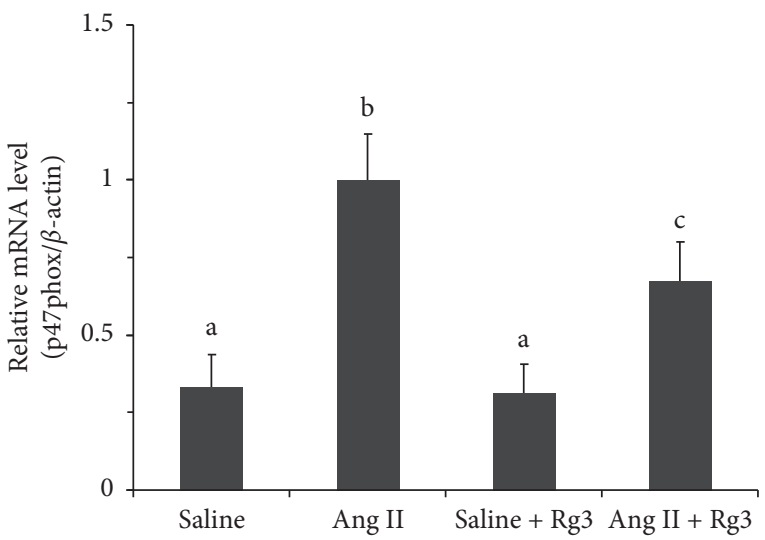

(d)

FIGURE 10: Levels of mRNA associated with inflammation, oxidative stress, and fibrosis in the renal tissue in mice. Relative mRNA levels of TNF- $\alpha$ (a), IL-6 (b), TGF- $\beta 1$ (c), and p47phox (d). GAPDH was used as a housekeeping gene. Data are presented as the mean \pm standard deviation, $n=4$. The same superscript letters indicate no significant difference between groups $(P>0.05)$; significant difference existed between groups that do not have a same superscript letter $(P<0.05)$.

associated with Ang II reduction in the myocardium [10]. Therefore, we aimed to assess whether the protective effects of Rg3 on the kidney are associated with Ang II reduction, and ACE2 levels are raised in the renal tissue.

Rg3, the principal component of Shenyi Capsule, is usually administered orally in clinical, and then it is metabolized by human intestinal bacteria. The metabolic product includes several kinds of ginsenosides [23, 24], such as ginsenoside Rh2 and protopanaxadiol. As Rg3 do not affect the kidneys directly, it is suitable to study the renal protective effects of Rg3 with animal experiments.

We carried out experiments in two models of nephropathy to test the hypothesis mentioned above. The rat model was at the compensation stage, with endogenous Ang II increase in the renal tissue, while the mouse model was at the decompensation stage, with exogenous increase of Ang II. These two experiments were mutually complemental.

The results obtained in both models were consistent. $\mathrm{Rg} 3$ reduced Ang II levels in the renal tissue in both models by up-regulating ACE2, and the mRNA levels of TNF- $\alpha$, IL6 , p47phox, and TGF- $\beta 1$ were down-regulated. These situations are similar to the previously reported [15], in which exogenous supplementary ACE2 downregulate Ang II levels.

In that research, upregulating ACE2 attenuated Ang II associated inflammation, oxidative stress and fibrosis in the renal tissue. Inflammatory cytokines such as TNF- $\alpha$, IL-6, IL$1 \beta$, and CCL-5 are down-regulated in renal tissue, the count of CD3 + cells was cut down. Dihydroethidium fluorescence showed that ACE2 reduced reactive oxygen species (ROS) in renal tissue, which mechanism was reversing NADPH Oxidase Activation. The syntheses of $\alpha$-smooth muscle actin $(\alpha$-SMA) and Collagen I \& III were reduced by downregulating TGF- $\beta 1$.

Because Rg3 upregulated ACE2 and downregulated TNF- $\alpha$, IL-6, p47phox, and TGF- $\beta 1$ as well as the research mentioned above, we determine that Rg3 attenuated Ang II associated inflammation, oxidative stress and fibrosis in the renal tissue. Meanwhile, Rg3 was able to reduce the progression of nephropathy in both models.

As there is no relevant report that could be retrieved, it seems that this research is the first to demonstrate that Rg3 upregulates tissue ACE2 levels in vivo. However, further investigation is needed to confirm that ACE2 upregulation 
in the renal tissue is the key mechanism of the renal protective effects of $\mathrm{Rg} 3$, and to reveal how $\mathrm{Rg} 3$ upregulates them. We might need to use other animals and models, for example, ACE2-knockout mice.

\section{Conclusion}

Overall, the current findings suggested Rg3 attenuates Ang IIassociated kidney injury and reduces the progression of nephropathy in animals. The probable mechanism is that $\mathrm{Rg} 3$ could reduce Ang II levels in the renal tissue by upregulating ACE2 and attenuate Ang II-induced inflammation, oxidative stress, and fibrosis in the renal tissue.

\section{Data Availability}

The data used to support the findings of this study are included within the article.

\section{Conflicts of Interest}

All the authors have no conflicts of interest to declare.

\section{Authors' Contributions}

Hui Liu and Yichuan Jiang have equally contributed to this work

\section{Acknowledgments}

This research was supported by the National Natural Science Foundation of China (Grant 81473378), Science and Technology Development Projects of Jilin Province (Grant 20170101002JC), and Outstanding Doctoral Cultivation Program of Norman Bethune Health Science Center of Jilin University (2014).

\section{Supplementary Materials}

Supplementary Figure 1: the chemical structure of $\mathrm{Rg} 3$. (Supplementary Materials)

\section{References}

[1] H. T. Vo, J. Y. Cho, Y. E. Choi, Y. S. Choi, and Y. H. Jeong, "Kinetic study for the optimization of ginsenoside Rg3 production by heat treatment of ginsenoside Rb1," Journal of Ginseng Research, vol. 39, no. 4, pp. 304-313, 2015.

[2] T. Xu, Z. Jin, Y. Yuan et al., "Ginsenoside Rg3 serves as an adjuvant chemotherapeutic agent and vegf inhibitor in the treatment of non-small cell lung cancer: a meta-analysis and systematic review," Evidence-Based Complementary and Alternative Medicine, vol. 2016, Article ID 7826753, 14 pages, 2016.

[3] E. H. Park, Y. J. Kim, N. Yamabe et al., "Stereospecific anticancer effects of ginsenoside Rg3 epimers isolated from heat-processed American ginseng on human gastric cancer cell," Journal of Ginseng Research, vol. 38, no. 1, pp. 22-27, 2014.

[4] M. Sun, Y. Ye, L. Xiao, X. Duan, Y. Zhang, and H. Zhang, "Anticancer effects of ginsenoside Rg3 (review)," International Journal of Molecular Medicine, vol. 39, no. 3, pp. 507-518, 2017.
[5] H. Yu, L. Teng, Q. Meng et al., "Development of liposomal Ginsenoside Rg3: formulation optimization and evaluation of its anticancer effects," International Journal of Pharmaceutics, vol. 450, no. 1-2, pp. 250-258, 2013.

[6] L. Li, J. Ni, M. Li et al., "Ginsenoside Rg3 micelles mitigate doxorubicin-induced cardiotoxicity and enhance its anticancer efficacy," Drug Delivery, vol. 24, no. 1, pp. 1617-1630, 2017.

[7] L. Q. Cheng, R. N. Ju, M. H. Bang, M. K. Kim, and D. C. Yang, "Conversion of major ginsenoside Rb1 to 20(S)-ginsenoside Rg3 by microbacterium sp. GS514," Phytochemistry, vol. 69, no. 1, pp. 218-224, 2008.

[8] L. H. Quan, J. W. Min, D. U. Yang, Y. J. Kim, and D. C. Yang, "Enzymatic biotransformation of ginsenoside Rb1 to 20(S)Rg3 by recombinant $\beta$-glucosidase from Microbacterium esteraromaticum," Applied Microbiology and Biotechnology, vol. 94, no. 2, pp. 377-384, 2012.

[9] L. P. Zhang, Y. C. Jiang, X. F. Yu et al., "Ginsenoside Rg3 improves cardiac function after myocardial ischemia/reperfusion via attenuating apoptosis and inflammation," EvidenceBased Complementary and Alternative Medicine, vol. 2016, Article ID 6967853, 8 pages, 2016.

[10] Y. Jiang, M. Li, Z. Lu et al., "Ginsenoside Rg3 induces ginsenoside Rb1-comparable cardioprotective effects independent of reducing blood pressure in spontaneously hypertensive rats," Experimental and Therapeutic Medicine, vol. 14, no. 5, pp. 4977-4985, 2017.

[11] Z. Jiuchang, B. Ratnadeep, G. Danny et al., "Angiotensinconverting enzyme 2 suppresses pathological hypertrophy, myocardial fibrosis, and cardiac dysfunction," Circulation, vol. 122, no. 7, pp. 717-728, 2010.

[12] H. Kobori, Y. Ozawa, Y. Suzaki, and A. Nishiyama, "Enhanced intrarenal angiotensinogen contributes to early renal injury in spontaneously hypertensive rats," Journal of the American Society of Nephrology, vol. 16, no. 7, pp. 2073-2080, 2005.

[13] L. Tang-Dong, Y. Xiao-Ping, L. Yun-He et al., "Role of inflammation in the development of renal damage and dysfunction in angiotensin II-induced hypertension," Hypertension, vol. 52, no. 2, pp. 256-263, 2008.

[14] Z. Liu, X. R. Huang, and H. Y. Lan, "Smad3 mediates ANG IIinduced hypertensive kidney disease in mice," American Journal of Physiology-Renal Physiology, vol. 302, no. 8, pp. F986-F997, 2012.

[15] J. Zhong, D. Guo, C. B. Chen et al., "Prevention of angiotensin II-mediated renal oxidative stress, inflammation, and fibrosis by angiotensin-converting enzyme 2," Hypertension, vol. 57, no. 2, pp. 314-322, 2011.

[16] V. J. Dias Da Silva, P. C. Cavalcante Viana, R. de Melo Alves, H. C. Salgado, N. Montano, and R. Fazan Jr., "Antihypertensive action of amiodarone in spontaneously hypertensive rats," Hypertension, vol. 38, no. 3, pp. 597-601, 2001.

[17] K. J. Livak and T. D. Schmittgen, "Analysis of relative gene expression data using real-time quantitative PCR and the 2(Delta Delta C(T)) method," Methods, vol. 25, no. 4, pp. 402-408, 2001.

[18] S. R. Tipnis, N. M. Hooper, R. Hyde, E. Karran, G. Christie, and A. J. Turner, "A human homolog of angiotensin-converting enzyme. Cloning and functional expression as a captopril-insensitive carboxypeptidase," Journal of Biological Chemistry, vol. 275, no. 43, pp. 33238-33243, 2000.

[19] G. Y. Oudit, G. C. Liu, J. C. Zhong et al., "Human recombinant ACE2 reduces the progression of diabetic nephropathy," Diabetes, vol. 59, no. 2, pp. 529-538, 2010. 
[20] J. Coresh, E. Selvin, L. A. Stevens et al., "Prevalence of chronic kidney disease in the United States," JAMA, vol. 298, no. 17, pp. 2038-2047, 2007.

[21] F. Paolo, "Prescribing angiotensin-converting enzyme inhibitors and angiotensin receptor blockers in chronic kidney disease," Nephrology, vol. 12, no. 1, pp. 81-89, 2007.

[22] K. Ki Sung, K. Hyun Young, Y. Noriko, P. Jeong Hill, and Y. Takako, "Preventive effect of 20(S)-ginsenoside Rg3 against lipopolysaccharide-induced hepatic and renal injury in rats," Free Radical Research, vol. 41, no. 10, pp. 1181-1188, 2007.

[23] B. Eunah, M. K. Han, P. Sunyoung, and K. Donghyun, "Metabolism of 20(S)- and 20(R)-ginsenoside Rg3 by human intestinal bacteria and its relation to in vitro biological activities," Biological \& Pharmaceutical Bulletin, vol. 25, no. 1, pp. 58-63, 2002.

[24] E. A. Bae, M. E. Han, and D. H. Kim, "Transformation of ginseng saponins to ginsenoside $\mathrm{Rh} 2$ by acids and human intestinal bacteria and biological activities of their transformants," Archives of Pharmacal Research, vol. 27, no. 1, pp. 61-67, 2004. 


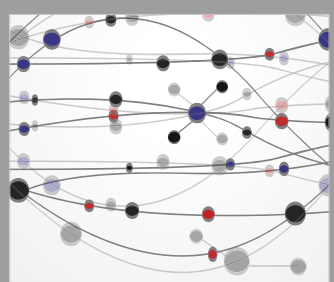

The Scientific World Journal
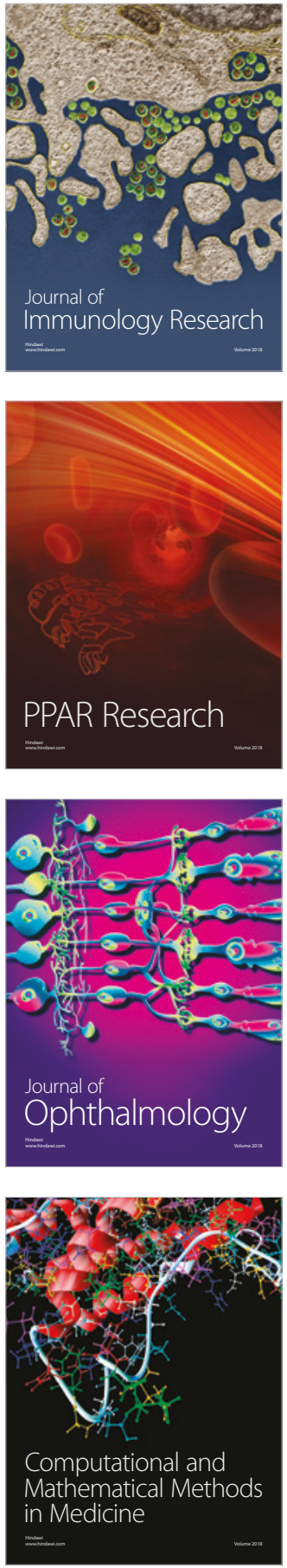

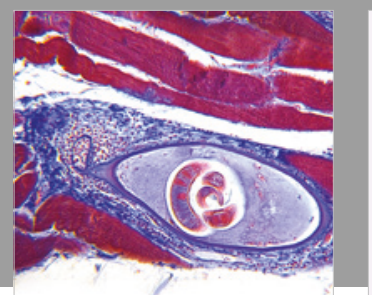

Gastroenterology Research and Practice

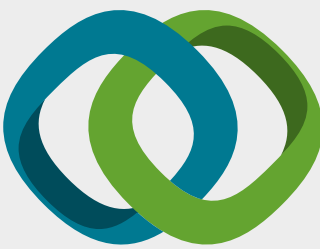

\section{Hindawi}

Submit your manuscripts at

www.hindawi.com
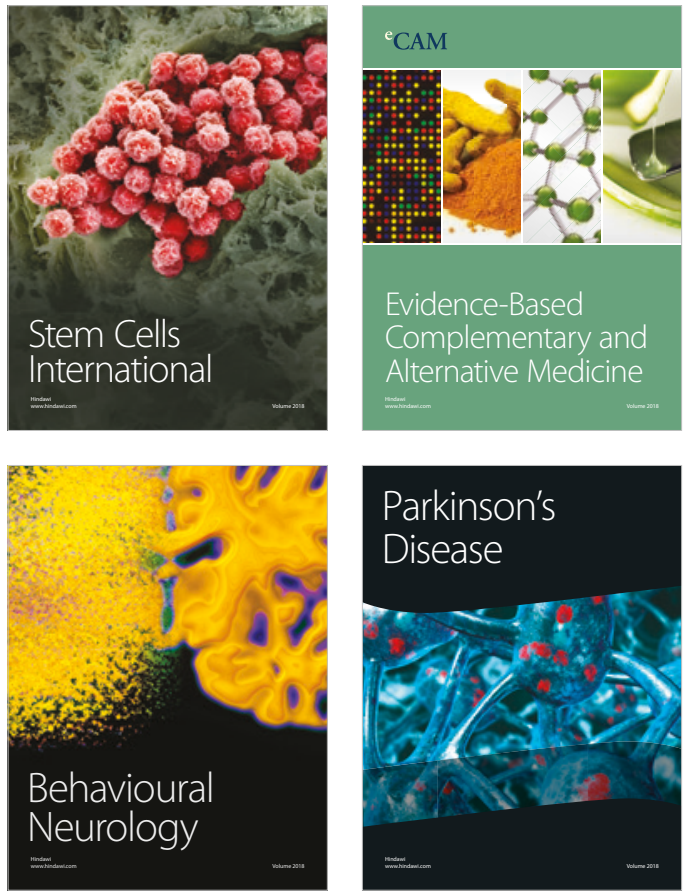

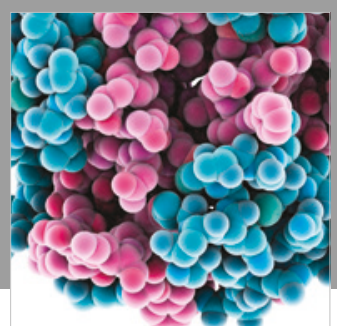

ournal of

Diabetes Research

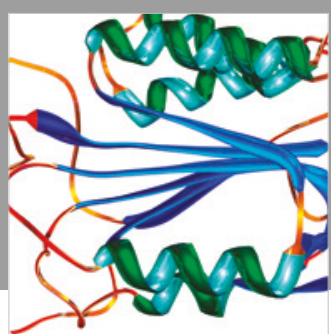

Disease Markers
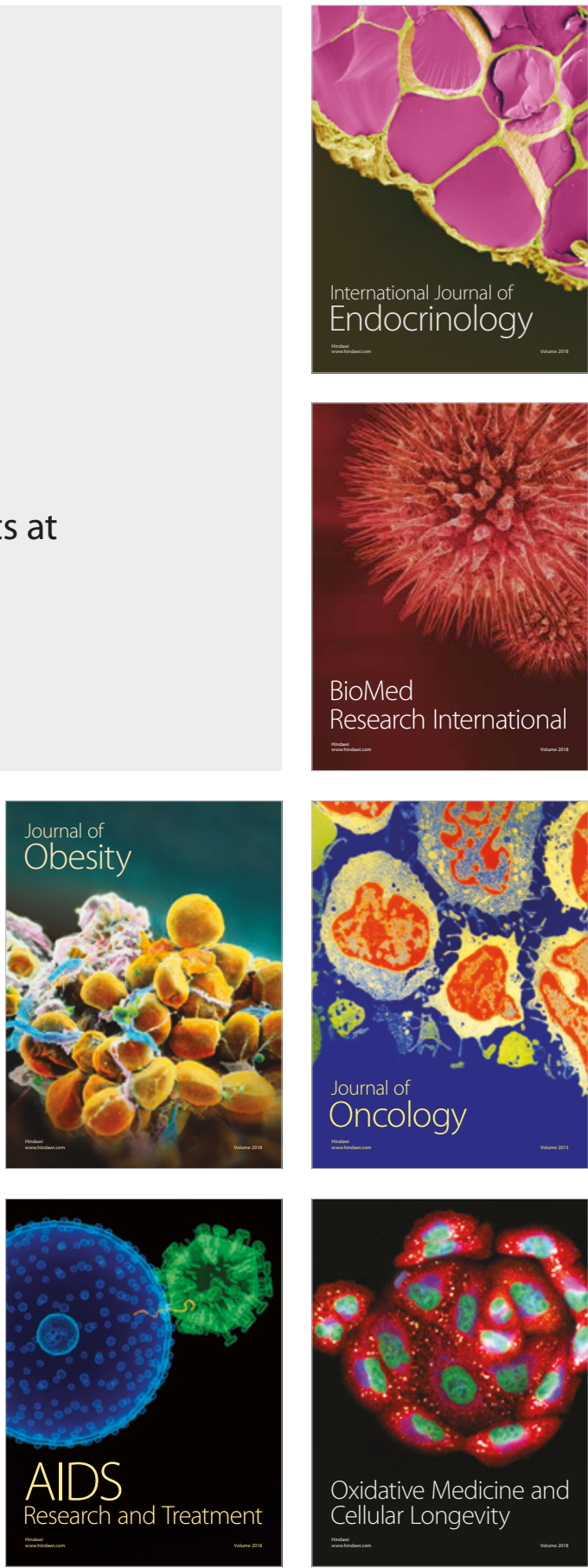